\title{
A novel approach to prevent spinal cord ischemia: Inoue stent graft with a side branch of small caliber for the reconstruction of the artery of Adamkiewicz
}

\author{
Takeshi Shimamoto, MD, ${ }^{\mathrm{a}}$ Akira Marui, MD, PhD, ${ }^{\mathrm{a}}$ Yoshimasa Nagata, ME, ${ }^{\mathrm{b}}$ Mitsuru Sato, ME, \\ Naritatsu Saito, MD, PhD, ${ }^{\mathrm{c}}$ Takahide Takeda, MD, ${ }^{\text {a }}$ Makiko Ueda, MD, PhD, ${ }^{\text {d }}$ Tadashi Ikeda, MD, PhD, ${ }^{\mathrm{a}}$ \\ Ryuzo Sakata, $\mathrm{MD}, \mathrm{PhD},{ }^{\mathrm{a}}$ and Kanji Inoue, $\mathrm{MD}^{\mathrm{b}, \mathrm{e}}$
}

\begin{abstract}
Objectives: Paraplegia remains a serious complication after endovascular repair of thoracic aortic aneurysms, and it has been reported that paraplegia might be due to ischemia of the artery of Adamkiewicz. This study investigates the feasibility of an Inoue stent graft with a side branch of a small caliber for the reconstruction of the intercostal artery branching the artery of Adamkiewicz.
\end{abstract}

\begin{abstract}
Methods: Branched Inoue stent grafts were implanted into the thoracic aorta and 11th intercostal artery of 5 mongrel dogs. The side branch measured $3 \times 5 \mathrm{~mm}$ and contained a bare-metal coronary stent for fixing to the intercostal arterial wall. Aortography and selective angiography of the 11th intercostal artery were performed before and immediately after implantation and after 1, 4, 8, and 12 weeks. The luminal diameter of the intercostal artery before implantation was $2.4 \pm 0.3 \mathrm{~mm}$.

Results: All stent grafts were successfully deployed. The main body of the graft did not develop endoleak or migrate, and the side branch remained patent for 12 weeks. Angiography performed 1 week postoperatively revealed smooth flow with slight stenosis $(4.2 \% \pm 1.7 \%)$ along the side branches and the intercostal arteries in all dogs. Four weeks postoperatively, however, mild concentric stenosis $(38 \% \pm 16 \%)$ along the side branch was observed in 4 of the 5 dogs. The percent stenosis at 8 and 12 weeks was $38 \% \pm 15 \%$ and $33 \% \pm 11 \%$, respectively; these values were not significantly different from the value at 4 postoperative weeks.
\end{abstract}

Conclusions: A novel Inoue stent graft with a side branch of small caliber was successfully deployed into the canine thoracic aorta and intercostal artery; the side branch remained patent for 12 weeks. This novel technique may enable endovascular reconstruction of the Adamkiewicz artery. (J Thorac Cardiovasc Surg 2010;139:655-9)

Endovascular aortic stent grafting is emerging as an alternative treatment strategy for thoracic and thoracoabdominal aortic aneurysm in selected high-risk patients. Even with a stent graft (SG), postoperative paraplegia remains a serious complication. Paraplegia might be caused by multiple factors; however, it is thought to be mainly due to spinal cord ischemia induced by the occlusion of the artery of Adamkiewicz (AA). Thus far, no report has been published on the endovascular reconstruction of an artery as small as an intercostal artery.

We previously reported the first successful implantation of a branched SG in patients with the following: aortic arch aneurysm secondary to type B aortic dissection with a tear imme-

\footnotetext{
From the Department of Cardiovascular Surgery, ${ }^{\text {a }}$ Kyoto University Graduate School of Medicine, Kyoto, Japan; PTMC Institute, ${ }^{b}$ Kyoto, Japan; Department of Cardiology, ${ }^{\mathrm{c}}$ Nagai Hospital, Mie, Japan; Department of Pathology, ${ }^{\mathrm{d}}$ Osaka City University Graduate School of Medicine, Osaka, Japan; and Department of Cardiovascular Surgery, ${ }^{\mathrm{e}}$ Shimabara Hospital, Kyoto, Japan.

Disclosures: Dr Kanji Inoue holds all patents of the Inoue stent graft. The Inoue stent graft was developed and made by Dr Kanji Inoue.

Received for publication Jan 20, 2009; revisions received March 19, 2009; accepted for publication May 15, 2009; available ahead of print Dec 9, 2009

Address for reprints: Takeshi Shimamoto, MD, Department of Cardiovascular Surgery, Kyoto University Graduate School of Medicine, 54 Shogoin Kawahara, Sakyo, Kyoto 606-8507, Japan (E-mail: shimamo@kuhp.kyoto-u.ac.jp). $0022-5223 / \$ 36.00$

Copyright (C) 2010 by The American Association for Thoracic Surgery doi:10.1016/j.jtcvs.2009.05.006
}

diately distal to the left subclavian artery, ${ }^{1}$ thoracoabdominal aneurysm involving the celiac axis, ${ }^{2}$ and suprarenal abdominal aortic aneurysm involving the superior mesenteric artery and both renal arteries. ${ }^{3}$ Since then, the safety and feasibility of branched SGs have been evaluated in a patient cohort of significant size with a sufficiently long follow-up period. ${ }^{4}$

In the present study, we have developed a novel Inoue SG with a side branch for the intercostal artery and examined the validity and feasibility of the grafting procedure by assessing the outcomes after graft implantation into the canine thoracic aorta.

\section{MATERIALS AND METHODS \\ Preparation of SGs}

The Inoue SG with a side branch of small caliber consists of a branched Dacron graft, detachable carrying and traction wires attached to the head and tail of the graft, a bare-metal coronary stent, and a looped balloon catheter (Velocity; Johnson \& Johnson, New Brunswick, NJ) sewn to the inside of the side branch by multiple sutures of 7-0 polypropylene, a $5 \mathrm{~F}$ guiding catheter, a $14 \mathrm{~F}$ introducer sheath, and a 0.014 -inch guidewire in the looped balloon catheter to select the intercostal artery (Figures 1 and 2).

The main body of the SG is externally supported by nickel-titanium rings, both ends of which are covered by the Dacron small tube to facilitate sealing function. The rings are self-expandable and folded with diameter-restricting ties at the insertion point to enable free movement of the graft in the thoracic aorta after the graft is released from the sheath. The diameter of the 


$$
\begin{aligned}
& \text { Abbreviations and Acronyms } \\
& \text { AA }=\text { artery of Adamkiewicz } \\
& \text { LDI }=\text { luminal diameter at implantation } \\
& \text { MLD }=\text { minimum lumen diameter } \\
& \text { SG }=\text { stent graft }
\end{aligned}
$$

body is approximately $14 \mathrm{~mm}$, which was designed to be $10 \%$ larger than the diameter of the canine thoracic aorta at the SG landing zone. Its length was $30 \mathrm{~mm}$.

The side branch was $5 \mathrm{~mm}$ long and $3 \mathrm{~mm}$ wide; the coronary stent was $18 \mathrm{~mm}$ long and $3 \mathrm{~mm}$ wide and was sewn to the base of the side branch with 7-0 polypropylene sutures. This 3-mm-wide stent stretches over the intercostal artery to maximally expand the branch portion of the graft and fix it in the appropriate position to the wall of the intercostal artery.

\section{Implantation of SGs}

Five adult mongrel dogs, weighing 18 to $20 \mathrm{~kg}$, were used in this experiment. The experimental procedures and handling of the animals were reviewed and approved by the Institutional Animal Care and Use Committee at the Medical School of Kyoto University. All procedures were in accordance with the "Guide for the Care and Use of Laboratory Animals" proposed by the Institute of Laboratory Animal Resources. After administration of general anesthesia with pentobarbital, the dogs were maintained on ventilatory support with $1 \%$ isoflurane in $100 \%$ oxygen.

First, the folded and compacted SG was introduced into the thoracic aorta after the systemic administration of heparin sodium, 100 units $/ \mathrm{kg}$, and the SG was advanced a few centimeters proximal to the intended position where the SG should be finally located. Second, the 0.014 -inch guidewire inserted through the looped balloon catheter selected the target intercostal artery. Then, the side branch with bare coronary stent was inserted into the intercostal artery along the guidewire by pulling back the whole folded SG, and the side branch and the coronary stent were dilated by inflation of a balloon catheter set inside. The SG main body was then unfolded and maximally dilated by balloon inflation to complete the expansion.

\section{Angiography}

Aortography and selective angiography of the 11th intercostal artery were performed under fluoroscopy with a contrast dye. The luminal diameters of the 11th intercostal artery and of the thoracic aorta in the proximity of the intercostal artery were measured before the insertion of the SG along with a scaled guidewire. Luminal diameter at implantation (LDI) was defined as the luminal diameter of the 11th intercostal artery immediately after implantation (Figure 3, A). Quantitative measurements of lesion length and minimum luminal diameter (MLD) of the stenotic lesions that emerged in the follow-up period and were located along the SG side branch were performed before the procedure, at the time of completion of the procedure, and at follow-up examinations (Figure 3, B). Independent quantitative analysis of intercostal angiography was performed by the staff at the Department of Cardiovascular Surgery, Kyoto University Hospital, in a blinded manner. Diameter stenosis was defined as (LDI - MLD)/LDI $\times 100$.

\section{Follow-up}

After implantation, $100 \mathrm{mg}$ ticlopidine and aspirin were administered daily. Follow-up angiography was postoperatively performed on weeks 1 , 4,8 , and 12 .

\section{Histology}

Each dog was humanely killed by a lethal injection of pentobarbital on week 12 . The thoracic aorta and 11th intercostal artery with the branched

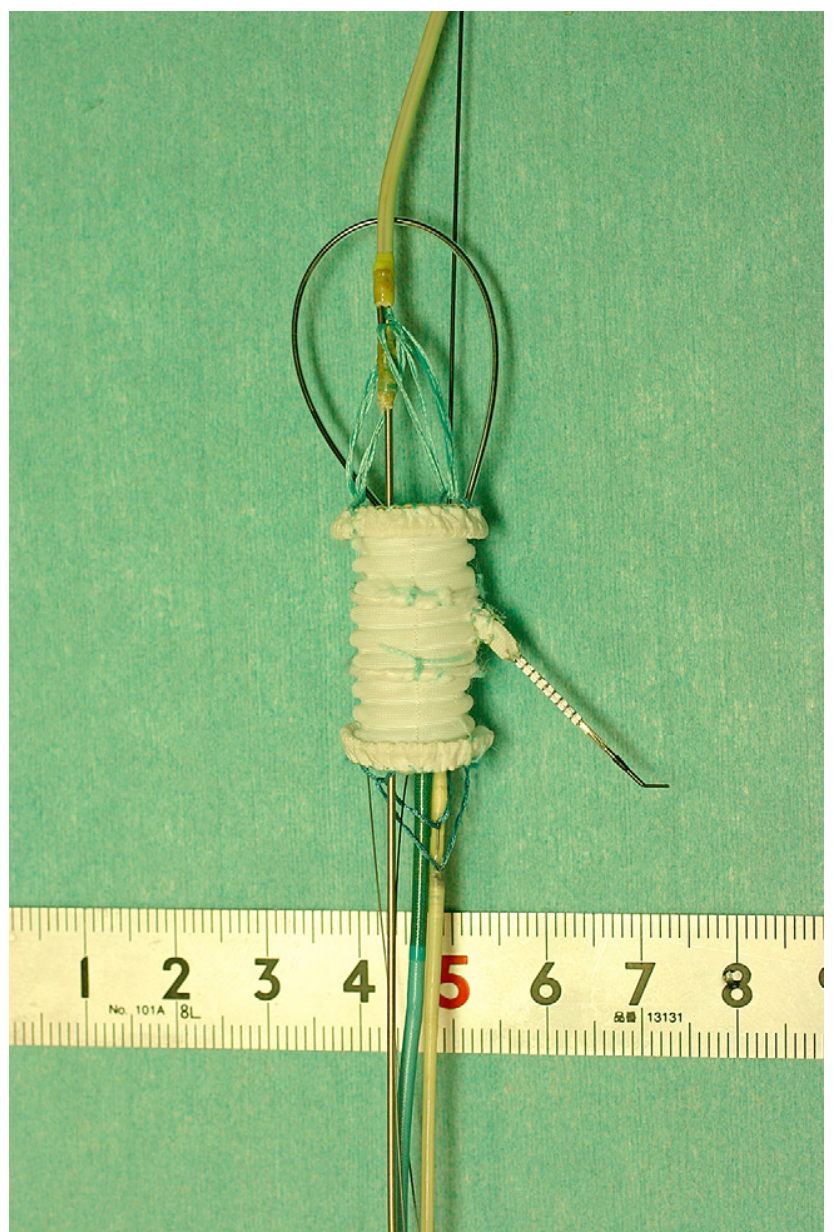

FIGURE 1. Inoue stent graft with a side branch of small caliber for implantation into the intercostal artery.

SG inside were perfusion-fixed with $10 \%$ buffered formalin and submitted for plastic embedding. The segments with the SG were cut longitudinally, and sections were stained with Cole's hematoxylin-eosin solution.

\section{Statistical Analysis}

All values are expressed as mean \pm standard deviation. Comparison of the two groups was performed using the Wilcoxon signed rank test (SPSS version 10; SPSS, Inc, Chicago, Ill).

\section{RESULTS \\ General Findings}

The SG was successfully deployed into the canine thoracic aorta and the 11th intercostal artery in all 5 dogs, without any instances of endoleak and stenosis along the main body, side branch, or intercostal artery. All coronary stents were successfully dilated inside the side branch of the SG and the intercostal artery by using nominal pressure. The time taken to complete the procedure was $66.2 \pm 14.4$ minutes (45-80 minutes), and the duration of intercostal artery occlusion was $13.0 \pm 1.6$ minutes (12-15 minutes). As our technical experience grew, both these times progressively decreased. 


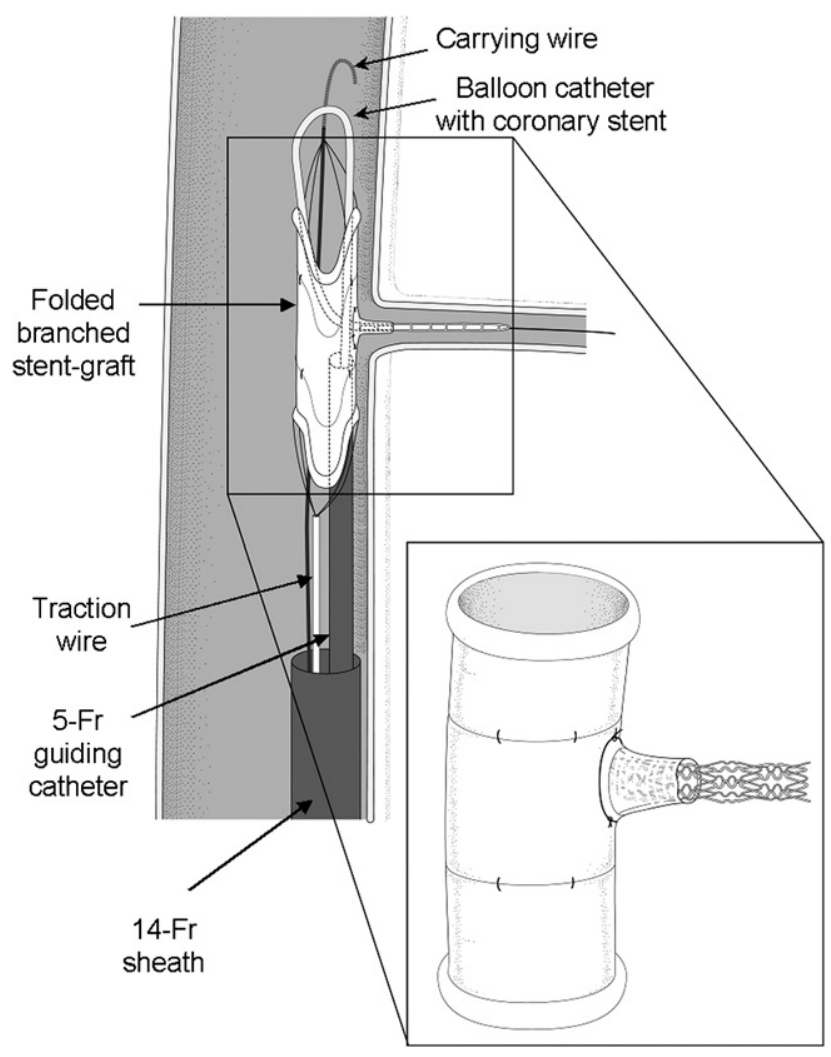

FIGURE 2. Schematic representation of the implantation of the branched Inoue stent graft into the canine thoracic aorta and intercostal artery.

There was no transient or permanent paraplegia or paraparesis. No procedure-related death occurred. One dog had a severe wound infection and died at 7 weeks after the operation, possibly of sepsis related to the wound infection. At autopsy, the SG did not show any abnormality, including infection or thrombotic occlusion.

\section{Angiography}

No unfavorable effects, including endoleak and migration that were attributable to the SG main body, were observed during the 12-week follow-up period. Before SG insertion, the luminal diameters of the 11th intercostal artery and of the thoracic aorta in the proximity of the intercostal artery were $2.43 \pm 0.30 \mathrm{~mm}$ and $12.7 \pm 0.93 \mathrm{~mm}$, respectively.

The MLD, lesion length, and diameter stenosis of the stent inside the side branch and the intercostal artery are shown in

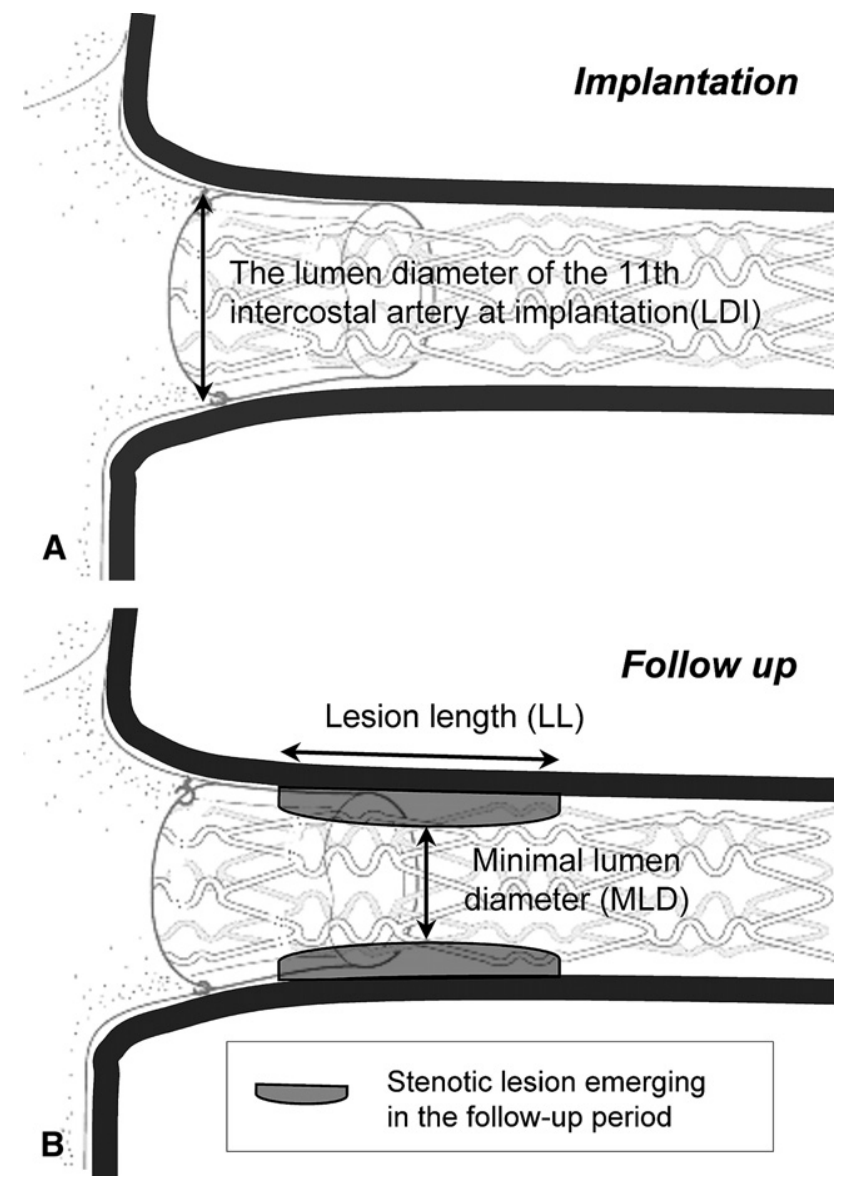

FIGURE 3. A and B, Schematic representation of LDI, MLD, and lesion length along the side branch of the stent graft.

Table 1. Smooth flow with no significant stenosis along the branch and intercostal artery was observed at 1 week postoperatively in all 5 dogs. At 4 weeks postoperatively, however, concentric mild stenosis along the midportion of coronary stent of the side branch appeared in 4 of the $5 \mathrm{dogs}$; nonetheless, very smooth blood flow along the side branch and intercostal artery was maintained. The degree of stenosis did not change significantly during the 12-week follow-up among the 4 surviving dogs (Figure 4).

\section{Histology}

Histologic examination showed that the SG completely adhered to the aortic wall. Both the main body and the

TABLE 1. Minimum luminal diameter $(M L D)$, lesion length $(L L)$, and diameter stenosis $(D S)$ at implantation and follow-up

\begin{tabular}{lccccc}
\hline & Implantation & 1 wk & 4 wk & 8 wk & 12 wk \\
\hline MLD (mm) & 3.0 & $2.9 \pm 0.1$ & $1.9 \pm 0.5^{*}$ & $1.9 \pm 0.4^{*}$ & $2.0 \pm 0.3^{*}$ \\
LL (mm) & 0 & $0.1 \pm 0.1$ & $3.6 \pm 1.7^{*}$ & $4.5 \pm 2.3^{*}$ & $4.0 \pm 2.2^{*}$ \\
DS (\%) & 0 & $4.2 \pm 1.7$ & $38 \pm 16^{*}$ & $38 \pm 14^{*}$ & $33 \pm 11^{*}$ \\
\hline
\end{tabular}

MLD and LL were those of stenotic lesions that emerge in the follow-up period along with the SG side branch according to angiography. Luminal diameter at implantation ( $L D I)$ was defined as the luminal diameter of the 11 th intercostal artery immediately after implantation, and DS was defined as (LDI - MLD)/LDI $\times 100 . * P<.01$ versus the values at implantation. 

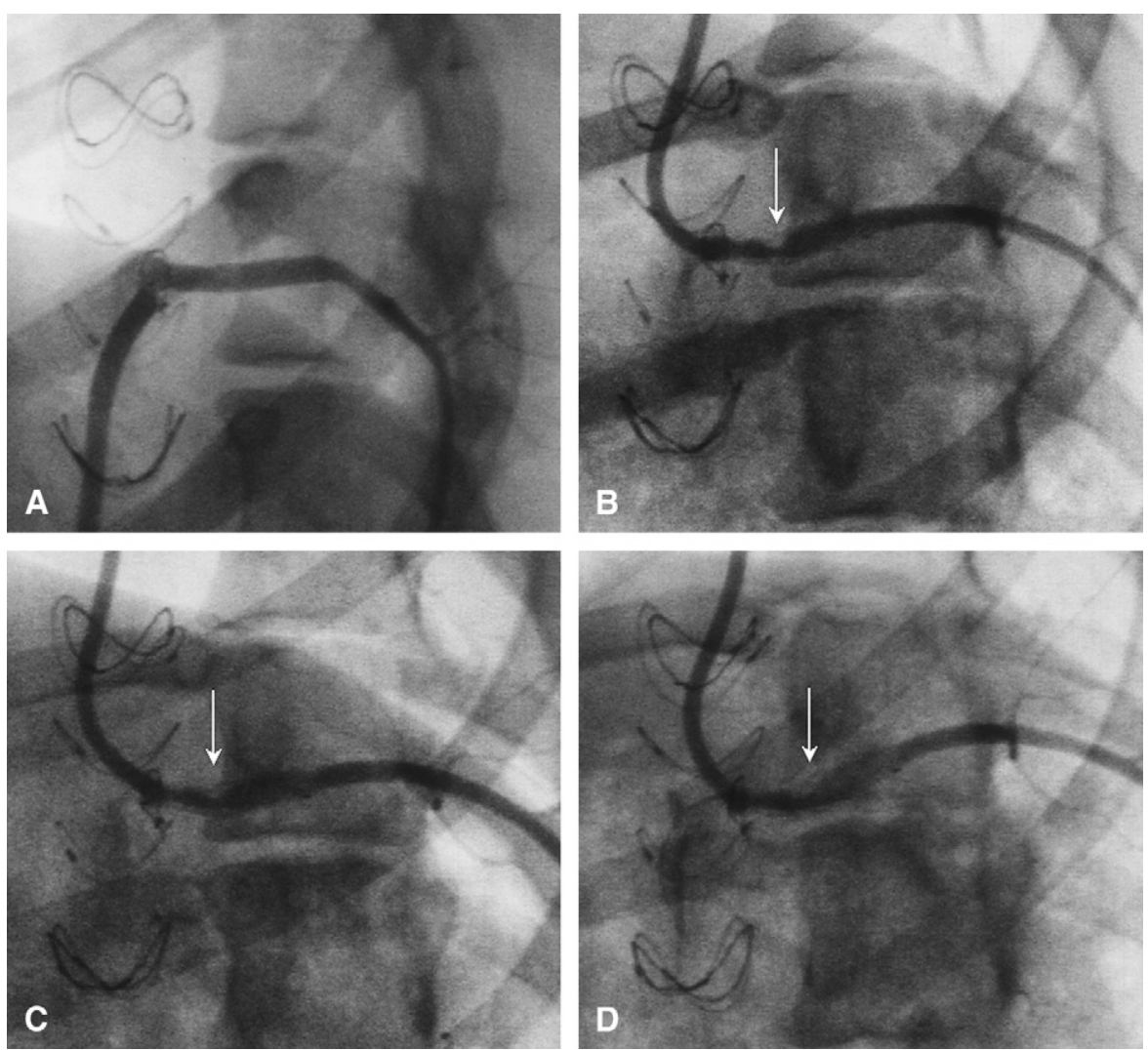

FIGURE 4. Representative angiographic images taken at 1 (A), 4 (B), 8 (C), and 12 (D) weeks after the operation. Note that moderate concentric stenosis appeared at 4 weeks after the operation and did not show any remarkable progression at 8 and 12 weeks after the operation (white arrows).

branch of the SG were covered by a thin layer of neointimal proliferation (Figure 5).

\section{DISCUSSION}

The main finding of this study is that the Inoue SG with a side branch of small caliber for the intercostal artery was deployable into the canine thoracic aorta, and its branch remained patent for 12 weeks. To the best of our knowledge, this is the first successful report of endovascular reconstruction of a small artery by using an SG with a side branch.

Postoperative paraplegia remains a serious complication even with the endovascular treatment of thoracic or thoracoabdominal aortic aneurysm. Its etiology is not yet fully understood; however, it has been reported that the risk of paraplegia increases if the AA is sacrificed. The recent advance of imaging modalities enables the accurate visualization of the AA. Therefore, the preoperative identification and subsequent endovascular reconstruction of the AA may significantly decrease the incidence of paraplegia, which improves the quality of life for the patients with this disease.

Previous reports have demonstrated that accessibility to the side branch in the target vessel is a significant problem with implantation of other types of branched SGs. ${ }^{5}$ In the Inoue SG, the large loop of the balloon catheter for the side branch effectively conducts an appropriate direction and longitudinal force along the axis of the side branch, which enables smooth insertion of the guidewire and the side branch into the target vessel. These characteristics make the implantation of the branched Inoue SG relatively simple..$^{1-4}$

The cause of the stenosis along the SG branch observed at 4 weeks after operation is unclear. Stenosis was remarkable only where the Dacron fabric was present outside the coronary stent, and the observed concentric narrowing was typical of neointimal hyperplasia. However, the degree of intimal proliferation along the branched portion was not severe, and this stenosis affected neither the short-term nor the long-term patency of the branch.

This study has several limitations. First, the anatomy of the thoracic aorta and the intercostal artery is not identical in humans and dogs. The normal diameter of the human thoracic aorta is approximately twice as large as that of the canine thoracic aorta. Moreover, the difference even increases if the canine aorta is compared with the human aneurysmal aorta. In fact, the diameter of the intercostal artery ${ }^{6}$ and the angle between the aorta and the intercostal artery are similar; thus, the technical difficulties in inserting the guidewire and side branch of the SG could be assumed to be similar. However, orientation of the device in a human tortuous and 


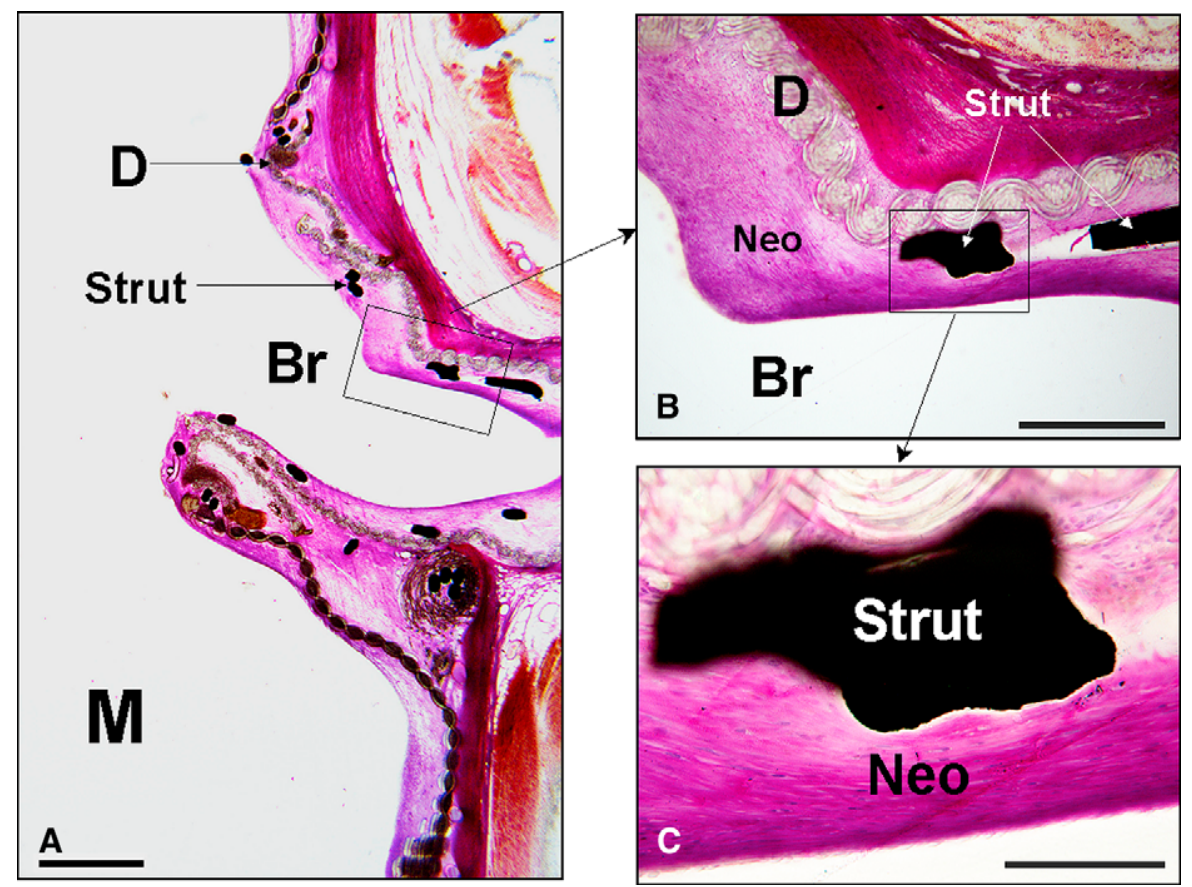

FIGURE 5. A, B, and C, Photomicrographs taken at 12 weeks after the implantation of the branched stent graft. Note that the branch portion of the stent graft is patent at 12 weeks after the operation and no thrombus is present. Mild neointimal proliferation can be seen around the stent graft. $M$, Lumen of the main body of the stent graft; $\mathrm{Br}$, lumen of the branch portion of the stent graft; Strut, stent strut; $D$, Dacron graft covering the stent graft; Neo, neointimal tissue proliferation. Scale bars: (A) $1 \mathrm{~mm}$, (B) $500 \mu \mathrm{m}$, and (C) $200 \mu \mathrm{m}$.

aneurysmal aorta can be much more complicated than the placement of the device in a normal undiseased vessel. Additionally, manipulation of the intercostal artery in a diseased aneurysmal aorta that may be lined with thrombus or other atherosclerotic debris can be much more challenging than doing so in a normal dog aorta. Second, an Inoue SG with a 3-mm-wide side branch was implanted into the normal canine thoracic aorta; the length of the side branch was $5 \mathrm{~mm}$. We did not assess the patency of longer side branches inserted into the intercostal artery that branched from the aneurysmal sac or of side branches narrower than $3 \mathrm{~mm}$; further, we did not evaluate the feasibility of the implantation of the other types of SGs. This was because side branches of different sizes may induce different postoperative pathophysiologic changes. Further investigation is necessary to verify the feasibility of the implantation of branched SGs with branches of different sizes and lengths in aneurysmal animal models or of implantation of side branches into narrower intercostal arteries.

In conclusion, we developed a novel Inoue SG with a side branch of small caliber and successfully deployed it into the canine thoracic aorta and intercostal artery. This novel technique may enable the endovascular reconstruction of the AA, and further studies should be conducted for its application in humans.

\section{References}

1. Inoue K, Sato M, Iwase T, Yoshida Y, Tanaka T, Tamaki S, et al. Clinical endovascular placement of branched graft for type B aortic dissection. J Thorac Cardiovasc Surg. 1996;112:1111-3.

2. Inoue K, Iwase T, Sato M, Yoshida Y, Ueno K, Tamaki S, et al. Transluminal endovascular branched graft placement for a pseudoaneurysm: reconstruction of the descending thoracic aorta including the celiac axis. J Thorac Cardiovasc Surg. 1997; 114:859-61.

3. Hosokawa H, Iwase T, Sato M, Yoshida Y, Ueno K, Tamaki S, et al. Successful endovascular repair of juxtarenal and suprarenal aortic aneurysms with a branched stent graft. J Vasc Surg. 2001;33:1087-92.

4. Saito N, Kimura T, Odashiro K, Toma M, Nobuyoshi M, Ueno K, et al. Feasibility of the Inoue single-branched stent-graft implantation for thoracic aortic aneurysm or dissection involving the left subclavian artery: short- to medium-term results in 17 patients. J Vasc Surg. 2005;41:206-12; discussion 212.

5. Roselli EE, Greenberg RK, Pfaff K, Francis C, Svensson LG, Lytle BW. Endovascular treatment of thoracoabdominal aortic aneurysms. J Thorac Cardiovasc Surg. 2007; $133: 1474-82$.

6. Malikov S, Rosset E, Paraskevas N, Magnan PE, Poirier M, Bartoli JM, et al. Extraanatomical revascularization of the artery of Adamkiewicz: anatomical study. Ann Vasc Surg. 2002;16:723-9. 